\title{
Reinforcement Learning for Autonomous Defence in Software-Defined Networking
}

\author{
Yi Han ${ }^{10000-0001-6530-4564]}$, Benjamin I.P. Rubinstein ${ }^{10000-0002-2947-6980]}$, Tamas Abraham ${ }^{2[0000-0003-2466-7646]}$, \\ Tansu Alpcan ${ }^{10000-0002-7434-3239]}$, Olivier De Vel ${ }^{2}$, Sarah Erfani ${ }^{10000-0003-0885-0643]}$, David Hubczenko ${ }^{2}$, \\ Christopher Leckie ${ }^{1[0000-0002-4388-0517]}$, and Paul Montague ${ }^{2}$ \\ 1 School of Computing and Information Systems, The University of Melbourne \\ 2 Defence Science and Technology Group \\ \{yi.han, benjamin.rubinstein, tansu.alpcan, sarah.erfani, caleckie\}@unimelb.edu.au \\ \{tamas.abraham, olivier.devel, david.hubczenko, paul.montague\}@dst.defence.gov.au
}

\begin{abstract}
Despite the successful application of machine learning (ML) in a wide range of domains, adaptabilitythe very property that makes machine learning desirable — can be exploited by adversaries to contaminate training and evade classification. In this paper, we investigate the feasibility of applying a specific class of machine learning algorithms, namely, reinforcement learning (RL) algorithms, for autonomous cyber defence in software-defined networking (SDN). In particular, we focus on how an RL agent reacts towards different forms of causative attacks that poison its training process, including indiscriminate and targeted, white-box and black-box attacks. In addition, we also study the impact of the attack timing, and explore potential countermeasures such as adversarial training.
\end{abstract}

Keywords: Adversarial reinforcement learning, Software-Defined Networking, cyber security, adversarial training.

\section{Introduction}

Machine learning has enjoyed substantial impact on a wide range of applications, from cyber-security (e.g., network security operations, malware analysis) to autonomous systems (e.g., decision-making and control systems, computer vision). Despite the many successes, the very property that makes machine learning desirable —adaptability —is a vulnerability to be exploited by an economic competitor or state-sponsored attacker. Attackers who are aware of the ML techniques being deployed can contaminate the training data to manipulate a learned ML classifier in order to evade subsequent classification, or can manipulate the metadata upon which the ML algorithms make their decisions and exploit identified weaknesses in these algorithm—so called Adversarial Machine Learning [13, 37, 18].

This paper focuses on a specific class of ML algorithms, namely, reinforcement learning (RL) algorithms, and investigates the feasibility of applying RL for autonomous defence in computer networks [14], i.e., the ability to "fight through" a contested environment—in particular adversarial machine learning attacks-and ensure critical services (e.g., email servers, file servers, etc.) are preserved to the fullest extent possible.

For example, consider a network as shown in Fig. 1 that consists of 32 nodes, one (node 3.8) of whom connects to the critical server, two (nodes 3.9 and 4.5) connect to potential migration destinations, and three (nodes 1.5, 2.7 and 3.6) connect to the attacker's hosts. The attacker aims to propagate through the network, and compromise the critical server. We aim to prevent this and preserve as many nodes as possible through the following RL approach:

- We first train two different types of RL agents: Double Deep Q-Networks (DDQN) [34] and Asynchronous Advantage Actor-Critic (A3C) [53]. The agents observe network states, and select actions such as "isolate", "patch", "reconnect", and "migrate". The agents gradually optimise their actions for different network states, based on the received rewards for maintaining critical services, costs incurred when shutting down non-critical services or migrating critical services.

- Once a working agent is obtained, we then investigate different ways by which the attacker may poison the training process of the RL agent. For example, the attacker can falsify part of the reward signals, or manipulate the states of certain nodes, in order to trick the agent to take non-optimal actions, resulting in either the critical server being compromised, or significantly fewer nodes being preserved. Both indiscriminate (untargeted) and targeted, white-box and black-box attacks are studied. 
- We also explore possible countermeasures—e.g., adversarial training — that make the training less vulnerable to causative/poisoning attacks.

- To make use of the developed capacity for autonomous cyber-security operations, we build our experimental platform around software-defined networking (SDN) [3], a next-generation tool chain for centralising and abstracting control of reconfigurable networks. The SDN controller provides a centralised view of the whole network, and is directly programmable. As a result, it is very flexible for managing and reconfiguring various types of network resources. Therefore, in our experiments the RL agents obtain all network information and perform different network operations via the SDN controller.

- Our results demonstrate that RL agents can successfully identify the optimal actions to protect the critical server, by isolating as few compromised nodes as possible. In addition, even though the causative attacks can cause the agent to make incorrect decisions, adversarial training shows great potential for mitigating the negative impact.

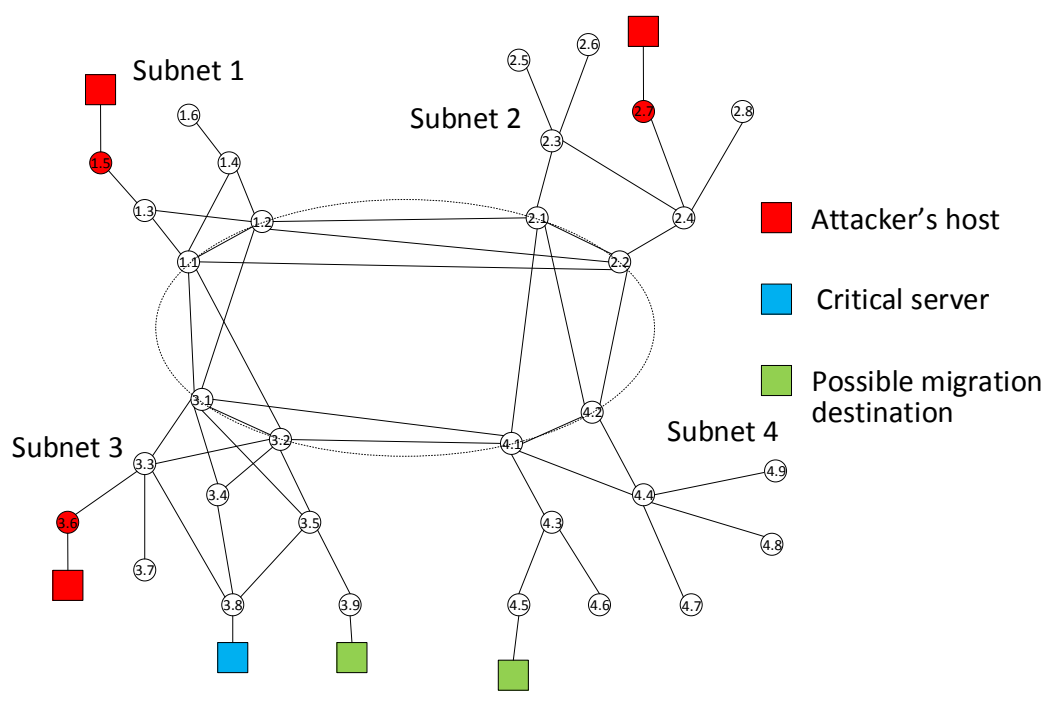

Fig. 1: An example network setup. The attacker propagates through the network to compromise the critical server, and the defender applies RL to prevent the critical server from compromise and to preserve as many nodes as possible.

The remainder of the paper is organised as follows: Section 2 briefly introduces the fundamental concepts in reinforcement learning and SDN; Section 3 defines the research problem; Section 4 introduces in detail the different forms of proposed attacks against RL; Section 5 presents the experimental results on applying RL for autonomous defence in SDN, and the impact of those causative attacks; Section 6 overviews previous work on adversarial machine learning (including attacks against reinforcement learning) and existing countermeasures; Section 7 concludes the paper, and offers directions for future work.

\section{Preliminaries}

Before defining the research problems investigated in this paper, we first briefly introduce the basic concepts in reinforcement learning and software-defined networking.

\subsection{Reinforcement Learning}

In a typical reinforcement learning setting [73], an agent repeatedly interacts with the environment: at each time step $t$, the agent (1) observes a state $s_{t}$ of the environment; (2) chooses an action $a_{t}$ based on its policy $\pi$-a mapping from 
the observed states to the actions to be taken; and (3) receives a reward $r_{t}$ and observes next state $s_{t+1}$. This process continues until a terminal state is reached, and then a new episode restarts from a certain initial state.

The agent's objective is to maximise its discounted cumulative rewards over the long run: $R_{t}=\sum_{\tau=t}^{\infty} \gamma^{\tau-t} r_{\tau}$, where $\gamma \in(0,1]$ is the discount factor that controls the trade-off between short-term and long-term rewards.

Under a given policy $\pi$, the value of taking action $a$ in state $s$ is defined as: $Q^{\pi}(s, a)=\mathbb{E}\left[R_{t} \mid s_{t}=s, a_{t}=a, \pi\right]$. Similarly, the value of state $s$ is defined as: $V^{\pi}(s)=\mathbb{E}\left[R_{t} \mid s_{t}=s, \pi\right]$. In this paper, we mainly focus on two widely cited RL algorithms: Double Deep Q-Networks (DDQN) [34] and Asynchronous Advantage Actor-Critic (A3C) [53].

Q-Learning Q-learning [73] approaches the above problem by estimating the optimal action value function $Q^{*}(s, a)=$ $\max _{\pi} Q^{\pi}(s, a)$. Specifically, it uses the Bellman equation $Q^{*}(s, a)=\mathbb{E}_{s^{\prime}}\left[r+\gamma \max _{a^{\prime}} Q^{*}\left(s^{\prime}, a^{\prime}\right)\right]$ to update the value iteratively. In practice, Q-learning is commonly implemented by function approximation with parameters $\theta: Q^{*}(s, a) \approx$ $Q(s, a ; \theta)$. At each training iteration $i$, the loss function is defined as: $L_{i}\left(\theta_{i}\right)=\mathbb{E}\left[\left(r+\gamma \max _{a^{\prime}} Q\left(s^{\prime}, a^{\prime} ; \theta_{i-1}\right)-\right.\right.$ $\left.\left.Q\left(s, a ; \theta_{i}\right)\right)^{2}\right]$.

Deep Q-Networks (DQN) Classic Q-learning networks suffer from a number of drawbacks, including (1) the i.i.d. (independent and identically distributed) requirement of the training data being violated as consecutive observations are correlated, (2) unstable target function when calculating Temporal Difference (TD) errors, and (3) different scales of rewards. Deep Q networks (DQN) [54] overcome these issues by (1) introducing experience replay, (2) using a target network that fixes its parameters $\left(\theta^{-}\right)$and only updates at regular intervals, and (3) clipping the rewards to the range of $[-1,1]$. The loss function for DQN becomes: $L_{i}\left(\theta_{i}\right)=\mathbb{E}\left[\left(r+\gamma \max _{a^{\prime}} Q\left(s^{\prime}, a^{\prime} ; \theta_{i}^{-}\right)-Q\left(s, a ; \theta_{i}\right)\right)^{2}\right]$.

Double DQN (DDQN) To further solve the problem of value overestimation, Hasselt et al. [34] generalise the Double Q-learning algorithm [33] proposed in the tabular setting, and propose Double DQN (DDQN) that separates action selection and action evaluation, i.e., one DQN is used to determine the maximising action and a second one is used to estimate its value. Therefore, the loss function is: $L_{i}\left(\theta_{i}\right)=\mathbb{E}\left[\left(r+\gamma Q\left(s^{\prime}, \arg \max _{a^{\prime}} Q\left(s^{\prime}, a^{\prime} ; \theta_{i}\right) ; \theta_{i}^{-}\right)-Q\left(s, a ; \theta_{i}\right)\right)^{2}\right]$.

Prioritised Experience Replay Experience replay keeps a buffer of past experiences, and for each training iteration, it samples uniformly a batch of experiences from the buffer, i.e., each experience is treated equally regardless of importance. Prioritised experience replay [70] assigns higher sampling probability to transitions that do not fit well with the current estimation of the $Q$ function. For DDQN, the error of an experience is defined as $\left|r+\gamma Q\left(s^{\prime}, \arg \max _{a^{\prime}} Q\left(s^{\prime}, a^{\prime} ; \theta\right) ; \theta^{-}\right)-Q(s, a ; \theta)\right|$. Experiences with larger errors are more likely to be chosen, and the errors will be updated at the end of each training iteration.

Asynchronous Advantage Actor-Critic (A3C) Mnih et al. [53] propose an asynchronous variant of the classical actor-critic algorithm, which estimates both the state value function $V\left(s ; \theta_{v}\right)$ and a policy $\pi\left(a \mid s ; \theta_{p}\right)$. Specifically, the asynchronous advantage actor-critic (A3C) algorithm uses multiple threads to explore different parts of the state space simultaneously, and updates the global network in an asynchronous way. In addition, instead of using discounted returns to determine whether an action is good, $\mathrm{A} 3 \mathrm{C}$ estimates the advantage function so that it can better focus on where the predictions are lacking.

\subsection{Software-Defined Networking}

In conventional online applications, the majority of the communication occurs directly between the client and the server. However, due to the wide use of content-delivery networks (CDN) and cloud services, it has become common for modern applications to access multiple servers and databases before data is finally returned to the client, resulting in a significantly higher proportion of "east-west" traffic. In addition, users nowadays may access an application with any type of device, e.g., smartphones, tablets and laptops, from anywhere around the world. These changes have contributed to an ever-increasing strain on traditional networks, largely due to conventional switches having their own control unit, making network reconfiguration time consuming. 
In order to better serve today's dynamic and high-bandwidth applications, a new architecture called SoftwareDefined Networking (SDN) has emerged [3]. There are three layers in the SDN architecture: (1) the application layer includes applications that deliver services. These applications communicate their network requirements to the controller via northbound APIs; (2) the SDN controller translates these requirements into low-level controls, and sends them through southbound APIs to the infrastructure layer; (3) the infrastructure layer comprises network switches that control forwarding and data processing.

One major advantage of SDN is that it decouples network control and forwarding functions, rendering the controller directly programmable. As a result, network resources can be conveniently managed, configured and optimised using standardised protocols. For example, Openflow [2] is a commonly-used protocol between the controller and the underlying switches. Note that in the simplest implementation, the controller follows a centralised design where it has a global view of the entire network. However, in order to manage large-scale networks, hierarchical and distributed designs can also be adopted.

There have been a number of proprietary and open-source SDN controller software platforms, such as Cisco's Open SDN Controller [5], Floodlight [6], NOX/POX [8] and Open vSwitch [4]. In this paper, we have opted to use OpenDaylight [49, 9], which is the largest open-source SDN controller today and which is updated regularly.

Applying RL in SDN One main challenge SDN faces arises from highly-dynamic traffic patterns, which motivate a requirement for the network to be reconfigured frequently. It has been demonstrated that RL is an ideal tool to accomplish such a task [69, 48, 46, 38, 77, 68, 51, 27, 40, 20]. For example, Salahuddin et al. [69] propose a roadside unit (RSU) cloud to enhance traffic flow and road safety. This RSU cloud is implemented using SDN, and leverages reinforcement learning to better cope with the dynamic service demands from the transient vehicles. In this way. the reconfiguration overhead can be minimised over the long run: increasing/decreasing the number of service centres, migrating services from one centre to another, for example. Mao et al. [48] design a job scheduling algorithm, called DeepRM, for computing clusters that have similar demands as SDN management. DeepRM uses colour images to represent system resources, e.g., CPU, RAM, as well as the resources required by each job slot, and exploits policy gradient methods to minimise average job slowdown.

\section{Problem Statement}

In this paper, we seek to answer the question: Can reinforcement learning be used for autonomous defence in SDN? We start with a scenario that does not consider the attacker poisoning the training process, and then investigate the impact of adversarial reinforcement learning. While we also briefly discuss potential countermeasures, we largely leave defences to future work.

\subsection{Reinforcement Learning Powered Autonomous Defence in SDN}

Consider a network of $N$ nodes (e.g., Fig. 11), $H=\left\{h_{1}, h_{2}, \ldots, h_{N}\right\}$, where $H_{C} \subset H$ is the set of critical servers to be protected (blue nodes in Fig. 11, $H_{M} \subset H$ is the set of possible migration destinations for $h \in H_{C}$ (green nodes), and $H_{A} \subset H$ is the set of nodes that have initially been compromised (red nodes). The attacker aims to propagate through the network, and penetrate the mission critical servers, while the defender/SDN controller monitors the system state, and takes appropriate actions in order to preserve the critical servers and as many non-critical nodes as possible.

Reflecting suggestions from past work, we consider a defender adopting RL. In this paper, we start with a simplified version, and make the following assumptions (Section 7 explains how they may be replaced): (1) each node (or link) only has two states: compromised/uncompromised (or on/off); (2) both the defender and the attacker know the complete network topology; (3) the defender has in place a detection system that can achieve a detection rate of $90 \%$, with no false alarms (before the causative attacks); (4) the attacker needs to compromise all nodes on the path (i.e., cannot "hop over" nodes).

Given these assumptions, in each step the defender:

1. Observes the state of the network-whether a node is compromised, and whether a link is switched on/off, e.g., there are 32 nodes and 48 links in Fig. 1. so one state contains an array of $800 s / 1 s$, where 0 means the node is uncompromised or the link is switched off, and 1 means the node is compromised or the link is switched on; 
2. Takes an action that may include: (i) isolating and patching a node; (ii) reconnecting a node and its links; (iii) migrating the critical server and selecting the destination; and (iv) taking no action. Note that, in this scenario, the defender can only take one type of action at a time, and if they decide to isolate/reconnect, only one node can be isolated/reconnected at a time. In the example of Fig. 11, there are $68(32+32+3+1)$ possible actions altogether;

3. Receives a reward based on (i) whether the critical servers are compromised; (ii) the number of nodes reachable from the critical servers; (iii) the number of compromised nodes; (iv) migration cost; and (v) whether the action is valid, e.g., it is invalid to isolate a node that has already been isolated, or to migrate a server to the current location.

Meanwhile, the attacker carefully chooses the nodes to compromise. For example, in the setting of Fig. 1, they infect a node only if it (1) is closer to the "backbone" network (nodes on the dashed circle); (2) is in the backbone network; or (3) is in the target subnet. Table 1 summarises this problem setting.

Table 1: Problem description: RL powered autonomous defence in SDN

\begin{tabular}{ll}
\hline \multicolumn{1}{c}{ Defender } & Attacker \\
\hline State & (1) Whether each node is compromised; \\
& \\
(2) Whether each link is turned on/off. & Compromise a node that satisfies certain conditions, $e . g .$, \\
Actions (1) Isolate and patch a node; & the node (1) is closer to the "backbone" network; (2) is \\
(2) Reconnect a node and its links; & \\
(3) Migrate the critical server and select the destination; & in the backbone network; or (3) in the target subnet. \\
(4) Take no action & Compromise the critical servers. \\
\hline Goals & $\begin{array}{l}\text { (1) Preserve the critical servers; } \\
\text { (2) Keep as many nodes uncompromised and reachable } \\
\text { from the critical servers as possible. }\end{array}$ \\
\end{tabular}

Alternatively, if we look from a game perspective, the problem can be defined as a two-player zero-sum security game [11] between the attacker $(A)$ and the defender $(D): G=\left\{P, A S^{i}, U^{i}, i \in\{A, D\}\right\}$, where $P=\{A, D\}, A S$ is the action set, and $U$ is the utility. Specifically, in the problem setting discussed here, while the defender can choose one of the four types of actions, the attacker has a pure/deterministic strategy (we will replace this and model the attacker as another agent [66] in future work). In addition, the defender's utility $U^{D}$ is the discounted accumulative rewards $R_{t}=\sum_{\tau=t}^{\infty} \gamma^{\tau-t} r_{\tau}, \gamma \in(0,1]$, and since it is a zero-sum game, $U^{A}=-U^{D}$. Considering tractability of computing equilibria (especially for much larger and more complex networks), we are interested in approximating the optimal response for the defender using RL algorithms.

\subsection{Causative Attacks against RL Powered Autonomous Defence System}

As an online system, the autonomous defence system continues gathering new statistics, and keeps training/updating its model. Therefore, it is necessary and crucial to analyse the impact of an adversarial environment, where malicious users can manage to falsify either the rewards received by the agent, or the states of certain nodes. In other words, this is a form of causative attack that poisons the training process, in order for the tampered model to take sub-optimal actions. In this paper, we investigate the two forms of attacks below.

1. Flipping reward signs. Suppose that without any attack, the agent would learn the following experience $\left(s, a, s^{\prime}, r\right)$, where $s$ is the current system state, $a$ is the action taken by the agent, $s^{\prime}$ is the new state, and $r$ is the reward. In our scenario, we permit the attacker to flip the sign of a certain number of rewards (e.g., $5 \%$ of all experiences), and aim to maximise the loss function of the RL agent. This is an extreme case of the corrupted reward channel problem [28], where the reward may be corrupted due to sensor errors, hijacks, etc.

2. Manipulating states. Again, consider the case where the agent learns an experience $\left(s, a, s^{\prime}, r\right)$ without any attack. Furthermore, when the system reaches state $s^{\prime}$, the agent takes the next optimal action $a^{\prime}$. The attacker is then allowed to introduce one false positive (FP) and one false negative (FN) reading in $s^{\prime}$, i.e., one uncompromised/compromised node is reported as compromised/uncompromised to the defender. As a result, instead of learning $\left(s, a, s^{\prime}, r\right)$, the agent ends up observing $\left(s, a, s^{\prime}+\delta, r^{\prime}\right)$ (where $\delta$ represents the FP and FN readings), and consequently may not take $a^{\prime}$ in the next step. 


\section{Attack Mechanisms}

This section explains in detail the mechanisms of the attacks introduced above.

\subsection{Attack I: Maximise Loss Function by Flipping Reward Signs}

Recall that the DDQN agent aims to minimise the loss function: $L_{i}\left(\theta_{i}\right)=\mathbb{E}\left[\left(r+\gamma Q\left(s^{\prime}, \arg \max _{a^{\prime}} Q\left(s^{\prime}, a^{\prime} ; \theta_{i}\right) ; \theta_{i}^{-}\right)-\right.\right.$ $\left.\left.Q\left(s, a ; \theta_{i}\right)\right)^{2}\right]$. In the $i^{t h}$ training iteration, $\theta_{i}$ is updated according to the gradient of $\partial L_{i} / \partial \theta_{i}$. The main idea for the first form of attack is to falsify certain rewards based on $\partial L_{i} / \partial r$, in order to maximise the loss $L_{i}$.

Specifically as shown in Algorithm 1, after the agent samples a batch of experiences for training, we calculate the gradient of $\partial L_{i} / \partial r$ for each of them, and flip the sign of experience with the largest absolute value of the gradient $\left|\partial L_{i} / \partial r\right|$ that satisfies $r \cdot \partial L_{i} / \partial r<0$ (if $r \cdot \partial L_{i} / \partial r>0$ flipping the sign decreases the loss function).

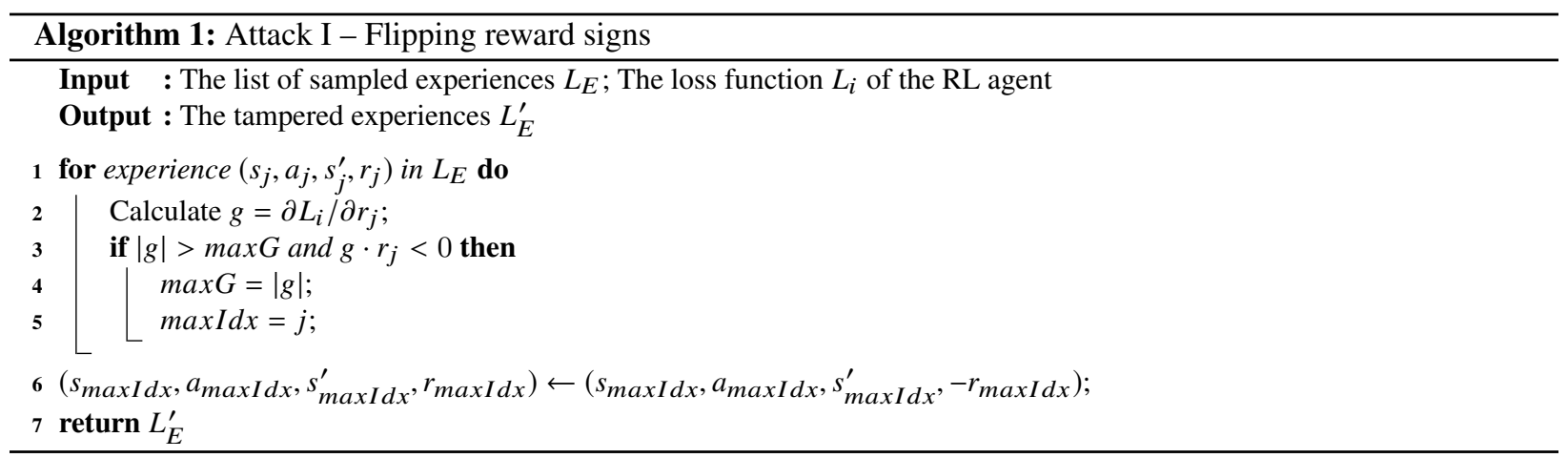

\subsection{Attack II: Prevent Agent from Taking Optimal/Specific Actions by Manipulating States}

Our experimental results show that the above form of attack is indeed effective in increasing the agent's loss function. However, it only delays the agent from learning the optimal actions. Therefore, the second form of attack directly targets the value function $Q$ (against DDQN agent) or the policy $\pi$ (against A3C agent).

1. Indiscriminate attacks. For each untampered experience $\left(s, a, s^{\prime}, r\right)$, indiscriminate attacks falsify the states of two nodes in the new state $s^{\prime}$, in order to prevent the agent from taking the next optimal action $a^{\prime}$ that has been learned so far (which may be different from the final optimal action for the given state), i.e., against DDQN agent the attacks minimise $\max _{a^{\prime}} Q\left(s^{\prime}+\delta, a^{\prime}\right)$, while against A3C agent the attacks minimise $\max _{a^{\prime}} \pi\left(a^{\prime} \mid s^{\prime}+\delta\right)$.

2. Targeted attacks. Targeted attacks aim to prevent the agent from taking a specific action (in our case, we find that this is more effective than tricking the agent to take a specific action). As an extreme case, this paper allows the attacker to know the (final) optimal action $a^{*}$ that the agent is going to take next ( $a^{*}$ may be different from $a^{\prime}$ ), and they seek to minimise the probability of the agent taking that action: for DDQN, the attacks minimise $Q\left(s^{\prime}+\delta, a^{*}\right)$; for A3C, the attacks minimise $\pi\left(a^{*} \mid s^{\prime}+\delta\right)$.

The details of the above two types of attacks are presented in Algorithm 2 (Note: Algorithm 2 is for the attacks against DDQN. Due to similarity, the algorithm for attacks against $\mathrm{A} 3 \mathrm{C}$ is omitted). In addition, we also consider the following variants of the attacks:

1. White-box attacks vs. Black-box attacks. In white-box attacks, the attacker can access the model under training to select the false positive and false negative nodes, while in black-box attacks, the attacker first trains surrogate model(s), and then uses them to choose the FPs and FNs. 
2. Limit on the choice of FPs and FNs. The above attacks do not set any limit on the choice of FPs and FNs, and hence even though the attacker can only manipulate the states of two nodes each time, overall, they still need to be able to control a number of nodes, which is not practical. Therefore, we first run unlimited white-box attacks, identify the top two nodes that have been selected most frequently as FPs and FNs respectively, and only allow the attacker to manipulate the states of those nodes.

3. Limit on the timing of the attack. The last type of attacks only introduces FPs and FNs in the first $m$ steps (e.g., $m=3$ ) in each training episode.

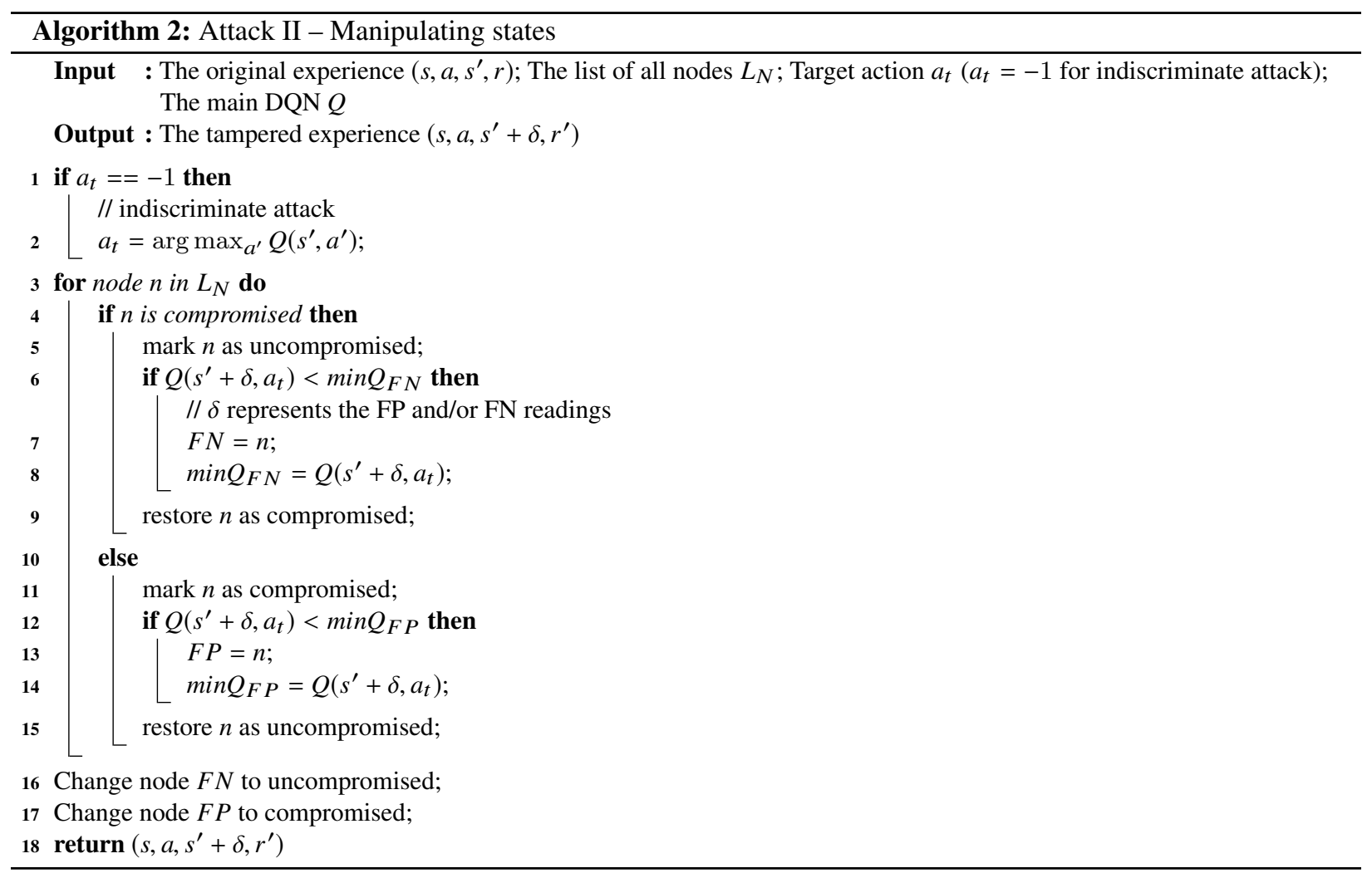

\section{Experimental Verification}

This section begins with a discussion of the experimental results obtained when applying RL to autonomous defence in a SDN environment without considering causative attacks. We then analyse the impact of the two forms of attacks explained in Section 4 Finally, we discuss adopting adversarial training as a potential countermeasure, and present some preliminary results. Experiments on causative attacks were performed on eight servers (equivalent to two Amazon EC2 t2.large instances and six t2.xlarge instances [1]), and each set of experiments was repeated 15 to 25 times.

\subsection{Autonomous Defence in a SDN}

For our experiments, as shown in Fig. 1. we created a network with 32 nodes and 48 links using Mininet [7], one of the most popular network emulators. OpenDaylight [49, 9] serves as the controller, and monitors the whole-ofnetwork status. The RL agent retrieves network information and takes appropriate operations by calling corresponding 
APIs provided by OpenDaylight. In the setup, the three nodes in red, i.e., nodes 1.5, 2.7 and 3.6, have already been compromised. Node 3.8 is the critical server to be protected, and it can be migrated to node 3.9 or 4.5 .

We trained a DDQN (with Prioritised Experience Relay) agent and an $\mathrm{A} 3 \mathrm{C}$ agent. We set the length of training such that the reward per episode for both agents reached a stable value well before training ended. The two agents learned two slightly different responses: the DDQN agent decides to first isolate node 3.6, then 1.3, 2.2 and finally 2.1, which means 21 nodes are preserved (see Fig. 2a); while the $\mathrm{A} 3 \mathrm{C}$ agent isolates nodes 1.5, 3.3, 2.2 and 2.1, keeping 20 nodes uncompromised and reachable from the critical server (see Fig. 2b.

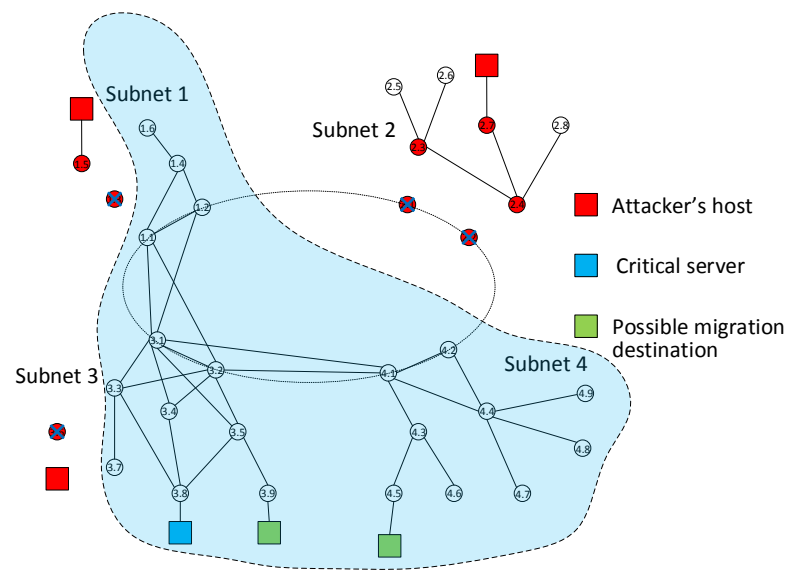

(a) DDQN

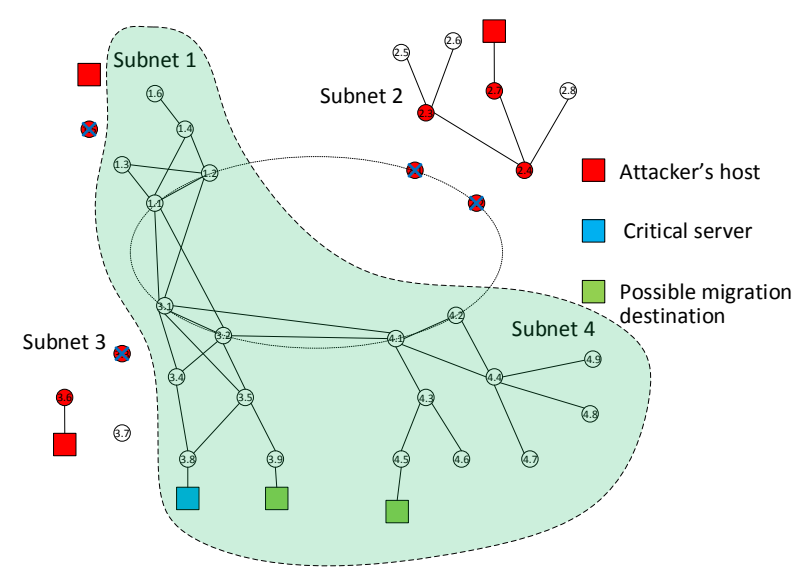

(b) $\mathrm{A} 3 \mathrm{C}$

Fig. 2: Optimal results without causative attacks (nodes in the blue/green shade are preserved)

\subsection{Attack I: Flipping Reward Sign}

This subsection presents the results of the first form of attack that flips the reward sign. In our experiments, we limit the total number of tampered experiences to $5 \%$ of all experiences obtained by the agent, and also set the number of tampered experiences per training iteration to the range of $[1,5]$.

As can be seen in Fig. 3, the attack is effective in increasing the agent's loss function. However, our results also suggest that this form of attack only delays the training as the agent still learns the optimal actions (although the delay can be significant).

\subsection{Attack II: Manipulate State-Indiscriminate Attacks}

Unlimited White-Box Attacks We start with unlimited indiscriminate white-box attacks, the case where the attacker has full access to the model under training. For each experience $\left(s, a, s^{\prime}, r\right)$ obtained by the agent, they can manipulate the states of any two nodes in $s^{\prime}$, i.e., one false positive and one false negative, in order to prevent the agent from taking the next optimal action $a^{\prime}$ that has been learned so far (note that it may be different from the final optimal action). Specifically, for the DDQN agent, the attacker minimises $\max _{a^{\prime}} Q\left(s^{\prime}+\delta, a^{\prime}\right)$; for the A3C agent, the attacker minimises $\max _{a^{\prime}} \pi\left(a^{\prime} \mid s^{\prime}+\delta\right)$.

Fig. 4 presents the results we obtained during our experiments. The leftmost bars in Figs. $4 \mathrm{a}$ and $4 \mathrm{~b}$ suggest that the unlimited indiscriminate white-box attacks are very effective against both DDQN and A3C. Specifically, the average number of preserved nodes decreases from 21 and 20 to 3.3 and 4.9, respectively. 


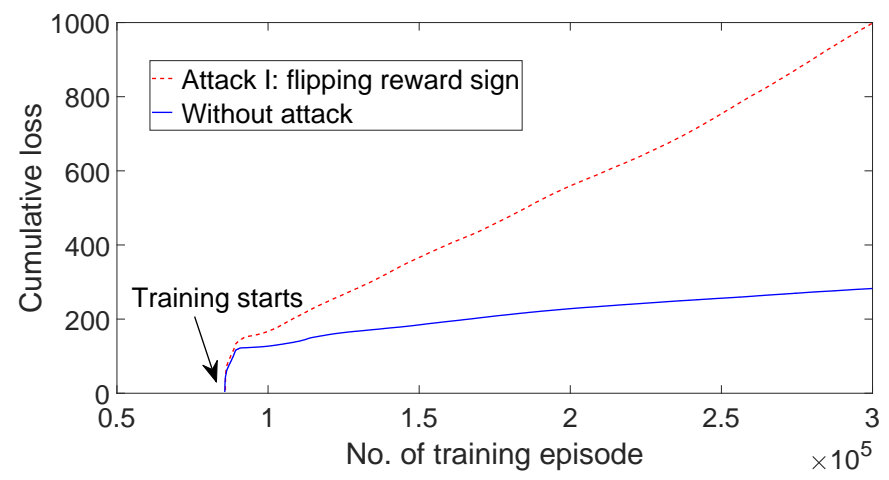

Fig. 3: Cumulative loss before and after flipping reward sign attacks (against DDQN)

White-Box Attacks with Limits on the Choices of False Positive and False Negative As pointed out in Subsection 3.2 even though the attacker only manipulates the states of two nodes each time, overall, they still need to be able to control a number of nodes, which is unlikely in practice. We calculate the number of times that each node is chosen in the above unlimited attacks, and find that some nodes are selected more frequently than others (Fig. 55, the histograms for the $\mathrm{A} 3 \mathrm{C}$ case are omitted due to similarity).

Therefore, when poisoning the DDQN agent, we limit the false positive nodes to $\{3.5$ (node ID 18), 4.1 (node ID 23) \}, and limit the false negative nodes to $\{2.7$ (node ID 12), 3.6 (node ID 19) $\}$. We use node 4.1 instead of 3.4 (node ID 17), as otherwise both selected nodes would be from the target subnet and directly connected to the target, which is unlikely in real situations. In the $\mathrm{A} 3 \mathrm{C}$ case, the false positive and false negative nodes are limited to $\{1.3$ (node ID 2), 3.5 (node ID 18) $\}$, and $\{2.7$ (node ID 12), 1.5 (node ID 4) $\}$, respectively.

The second and third bars in Figs. $4 \mathrm{a}$ and $4 \mathrm{~b}$ show that the limit has an obvious negative impact on the attack, especially the limit on the false negative nodes. Still, less than half of the nodes can be preserved on average, compared with the scenarios without attacks.

Black-Box Attacks with Limits on the Choices of False Positive and False Negative Nodes In our black-box attacks (both intra- and cross-models), the attacker does not have access to the training model. Instead, they train their own agents first, and use the surrogate models to poison the training of the target models by choosing false positive and false negative nodes. Specifically, we have trained a few DDQN and A3C agents with a different number of hidden layers from the target model, and observed that these surrogates can still prevent the critical server from compromising.

As illustrated by the rightmost two bars in Figs. $4 \mathrm{a}$ and $4 \mathrm{~b}$, black-box attacks are only slightly less effective than the counterpart white-box attacks despite the surrogate using a different model. This lends support that transferability also exists between RL algorithms, i.e., attacks generated for one model may also transfer to another model.

\subsection{Attack II: Manipulate State-Targeted Attacks}

In the targeted attacks considered here, the attacker is assumed to know the sequence of final optimal actions, and attempts to minimise the probability of the agent following that sequence. It should be pointed out that we have also studied the case where the attacker instead maximises the probability of taking a specific non-optimal action for each step, but our results suggested that this is less effective.

We find that in targeted attacks, certain nodes are also selected more frequently as a false positive and false negative. In this scenario, we limit false positive nodes to (1) $\{3.5$ (node ID 18), 4.1 (node ID 23) $\}$ against DDQN, (2) \{2.6 (node ID 11), 1.4 (node ID 3) $\}$ against A3C, and limit false negative nodes to (1) $\{1.5$ (node ID 4), 2.1 (node ID 6) $\}$ against DDQN, (2) $\{4.1$ (node ID 23), 2.4 (node ID 9) $\}$ against A3C.

Our results, summarised in Fig. 6 indicate that (1) compared with the results on indiscriminate attacks, targeted attacks work better, especially against DDQN (fewer nodes are preserved on average), as the attacker is more knowl- 


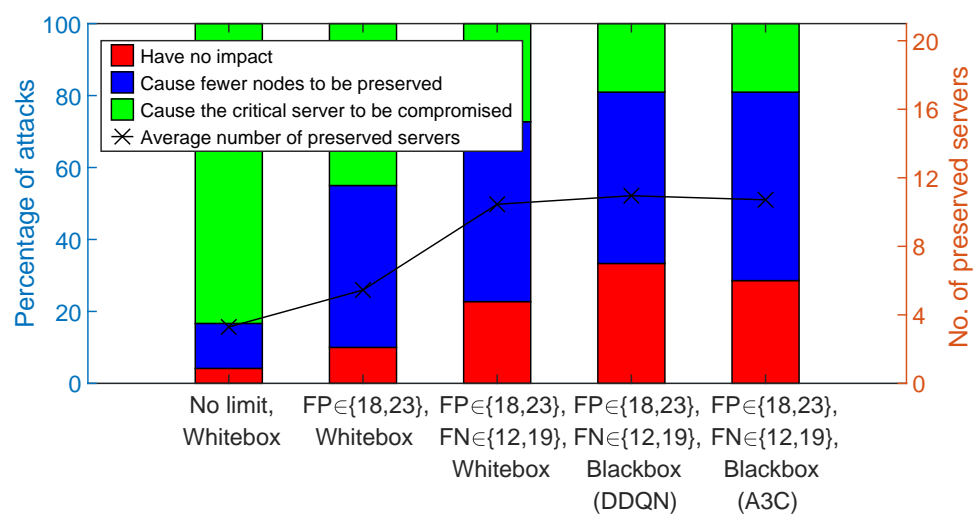

(a) Indiscriminate attacks against DDQN

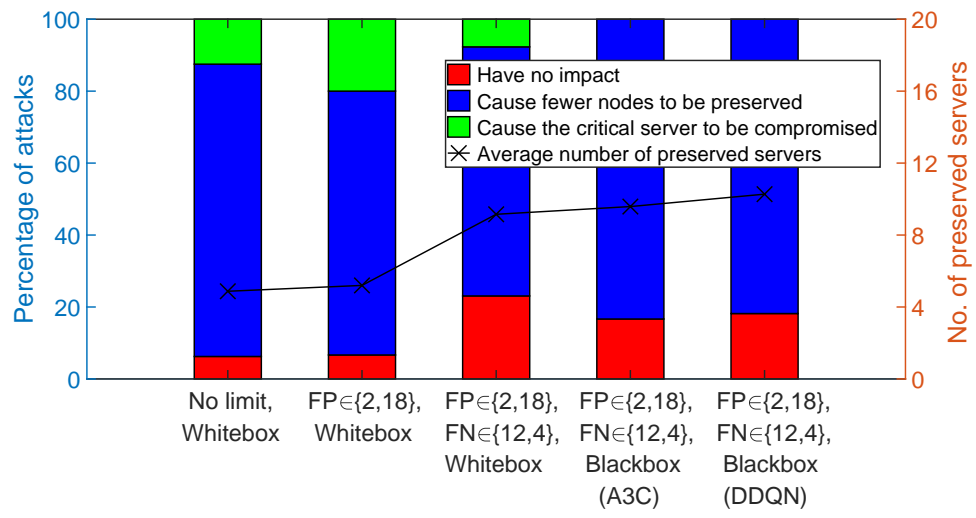

(b) Indiscriminate attacks against $\mathrm{A} 3 \mathrm{C}$

Fig. 4: Indiscriminate attacks against DDQN \& A3C. The bars indicates the percentage of attacks (left $y$-axis) that (1) have no impact; (2) cause fewer nodes to be preserved; and (3) cause the critical server to be compromised. The line marked by " $~ "$ " indicates the average number of preserved servers (right $y$-axis). The five types of attacks are: (1) white-box, no limit on FNs\&FPs; (2) white-box, with limits on FP but not on FN, (3) white-box, with limits on both FP and FN; (4) black-box, same algorithm, with limits on both FPs and FNs; (5) black-box, different algorithm, with limits on both FPs and FNs.

edgeable in this case; (2) similar to the indiscriminate case, black-box attacks achieve comparable results to the whitebox attacks, further demonstrating the transferability between DDQN and A3C.

\subsection{Timing Limits for the Attacks}

The attacks discussed so far allowed the attacker to poison every experience obtained by the agent. A possible limitation on this assumption is to examine whether these attacks can remain successful when the attacker can only manipulate part of the experiences. Therefore, in this subsection we shall look at attacks that poison only a subset (the first three steps) in each training episode.

Figs. $7 \mathrm{a}$ and $7 \mathrm{~b}$ depict the results of the time-limited version of (cross-model) black-box attacks against DDQN and white-box attacks against A3C (both with limit on the choices of FPs and FNs), respectively. The results suggest that even though the time limit has a negative impact in every scenario studied, the attacks are still effective. 


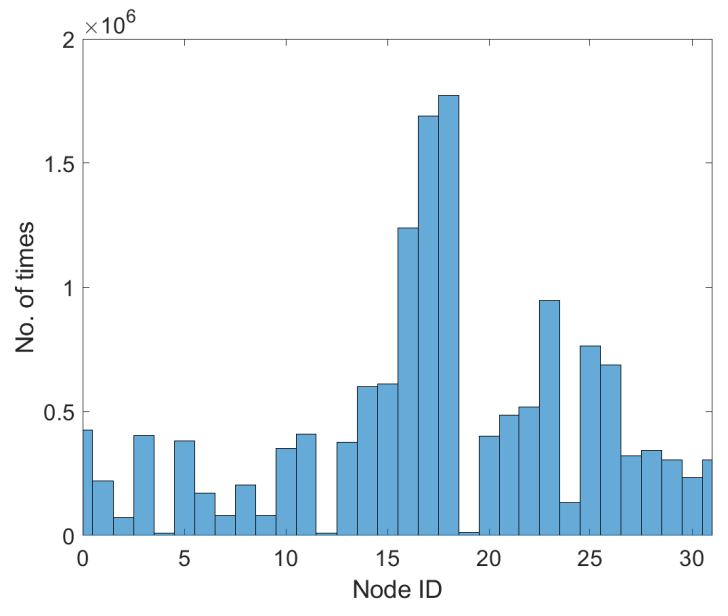

(a) False positive

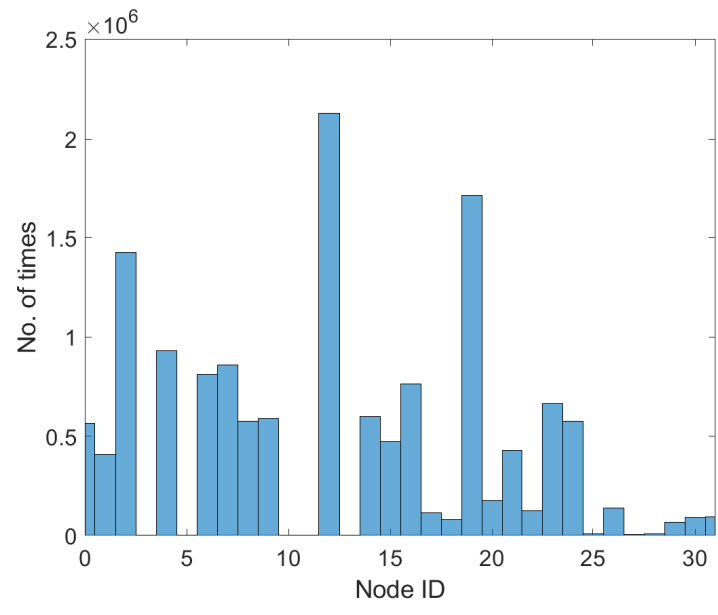

(b) False negative

Fig. 5: Histograms of the false positive and false negative nodes being selected against DDQN. N.B.: The node IDs $\{0,1, \ldots, 31\}$ are ordered and mapped to the node sequence $\{1.1,1.2, \ldots, 1.6,2.1, \ldots, 2.8,3.1, \ldots, 3.9,4.1, \ldots, 4.9\}$

\subsection{Discussion on Countermeasures}

In supervised learning problems, adversarial training [74, 31, 75] has the defender select a target point $(x, y)$ from the training set, modify $x$ to $x^{*}$ (i.e., generates an adversarial sample), and then inject $\left(x^{*}, y\right)$ back into the training set, under the implicit assumption that the true label $y$ should not change given the instance has been only slightly perturbed.

In our RL setting, while the attacker manipulates the observed states to minimise the probability of the agent taking the optimal action $a$ in state $s$, the defender can construct adversarial samples that counteract the effect. For example, for each experience $\left(s, a, s^{\prime}, r\right)$, the defender can increase $r$ by a small amount, e.g., 5\% of the original value, given that $r$ is positive and $a$ is not chosen randomly (the probability of choosing an action randomly decreases as the training proceeds). The rationale behind this modification is that when the poisoning attack starts out, it is likely that $a$ is still the optimal action (that has been learned so far) for state $s$. If $r$ is positive it means that action $a$ is a relatively good option for $s$, and since the attacker has poisoned the state to prevent the agent from taking $a$, we slightly increase $r$ to encourage the agent to take action $a$.

We have tested the above idea against indiscriminate white-box attacks with a limit on the choices of FPs and FNs against DDQN. Specifically, for each experience $\left(s, a, s^{\prime}, r\right)$ whose $r$ is positive and $a$ is not selected randomly, we change it to $\left(s, a, s^{\prime}, \min (1.0,1.05 r)\right)$. Note that our experimental results suggest that adding $5 \%$ error to the reward signal when there is no attack will not prevent the agent from learning the optimal actions, although it may cause some delay. The results in Fig. 8 indicate that adversarial training can make the training process much less vulnerable.

However, the results are still preliminary, and we plan to further investigate other forms of adversarial training. For example, Pinto et al. [66] model all potential disturbances as an extra adversarial agent, whose goal is to minimise the discounted reward of the leading agent. They formulate the policy learning problem as a two player zero-sum game, and propose an algorithm that optimises both agents by alternating learning one agent's policy with the other policy being fixed. In addition, we will also study the impact of the loss function, prioritised experience replay, ensemble adversarial training [75] and other, more intrusive types of attacks, where the adversary is aware of the defence method, and attacks the defended model.

\section{Related Work}

This section reviews ways in which attackers can target machine learning systems and current defence mechanisms. We first present a taxonomy on attacks against (primarily) supervised classifiers, and then summarise recent work 


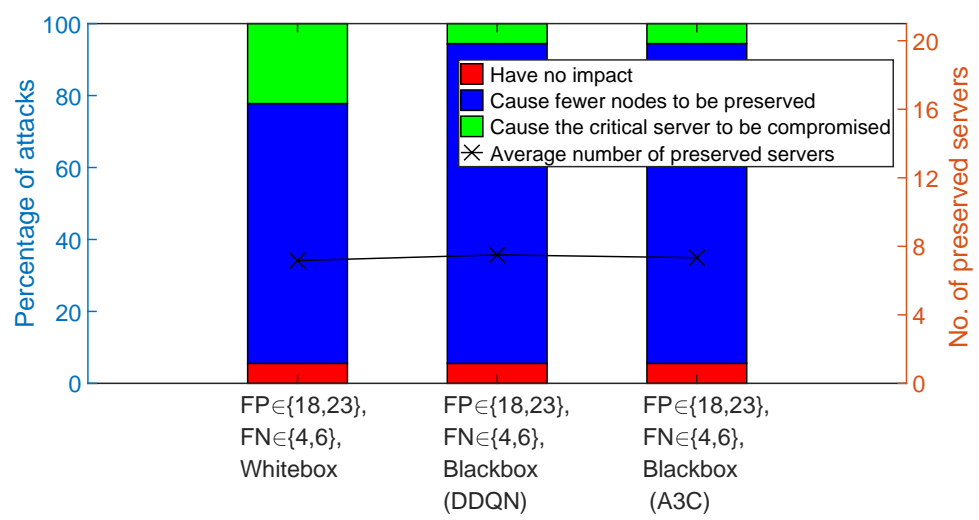

(a) Targeted attacks against DDQN

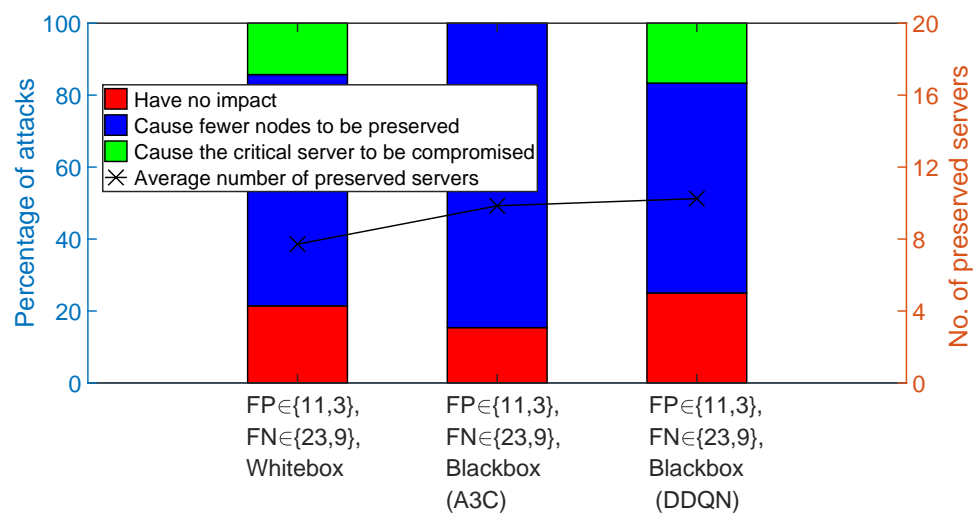

(b) Targeted attacks against A3C

Fig. 6: Targeted attacks against DDQN \& A3C. The bars indicate the percentage of attacks (left $y$-axis) that (1) have no impact; (2) cause fewer nodes to be preserved; and (3) cause the critical server to be compromised. The line marked by " $\times$ " indicates the average number of preserved servers (right $y$-axis).

that applies/modifies these attacks to manipulate RL systems. Finally, we review existing countermeasures against adversarial machine learning.

\subsection{Taxonomy of Attacks against Machine Learning Classifiers}

Barreno et al. [13] develop a qualitative taxonomy of attacks against machine learning classifiers based on three axes: influence, security violation and specificity.

- The axis of influence concerns the attacker's capabilities: in causative attacks, the adversary can modify the training data to manipulate the learned model; in exploratory attacks, the attacker does not poison training, but carefully alters target test instances to flip classifications. The resulting malicious instances which resemble legitimate data are called adversarial samples.

- The axis of security violation indicates the consequence desired by the attacker: integrity attacks are an example of deception attacks (achieving uncertainty, incompleteness, etc.), resulting in indecision, delayed decisions, poor or even wrong decisions, in decision-making systems. In this type of attack, the malicious instances bypass the filter as false negatives. In contrast, availability attacks seek to cause a denial-of-service against legitimate instances.

- The axis of specificity refers to the target of the attacks: indiscriminate attacks aim to degrade the classifier's performance overall, while targeted attacks focus on a specific type of instance, or a specific instance. 


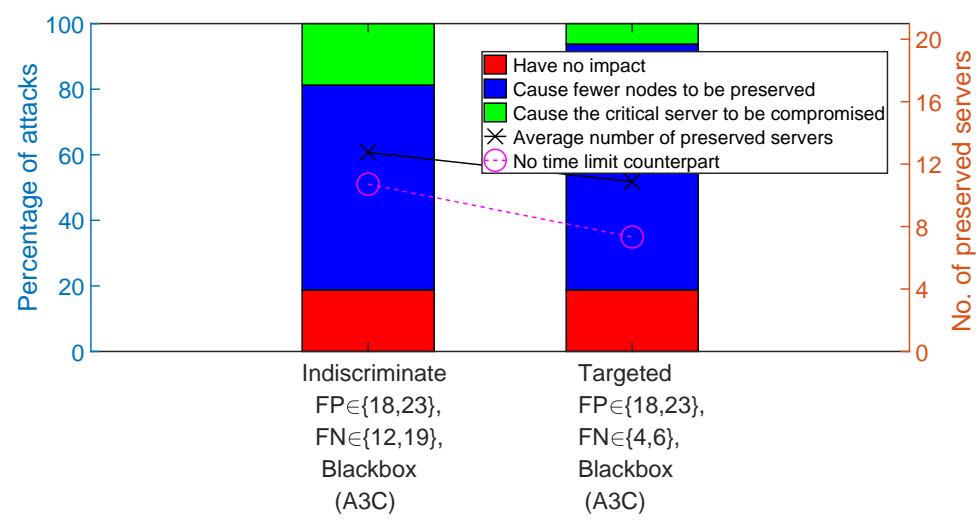

(a) Time limited cross-model black-box attacks against DDQN

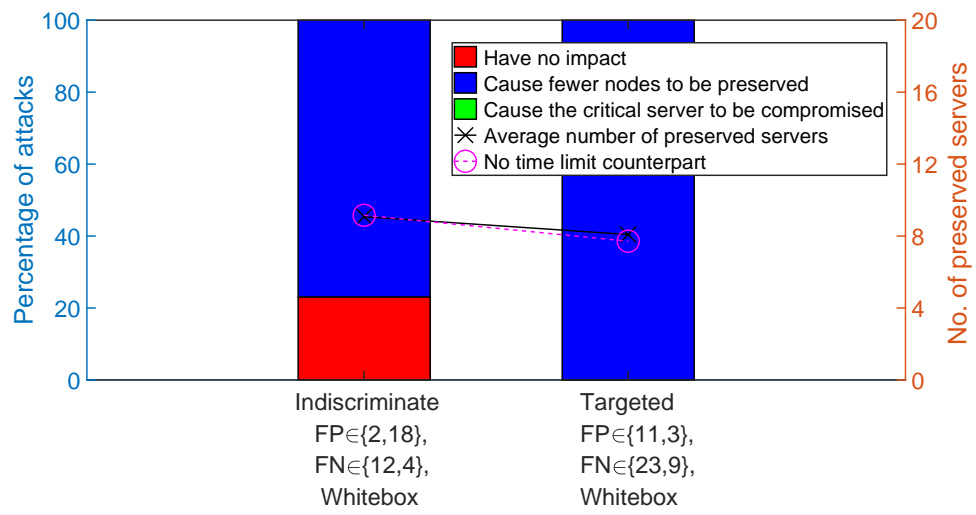

(b) Time limited white-box attacks against A3C

Fig. 7: Attacks against DDQN\&A3C with time limit. The attacker only poisons the first three steps per training episode.

Table 2 uses the taxonomy to classify previous work on adversarial machine learning against classifiers. As can be seen, more focus has been paid to exploratory integrity attacks. Presently, the Fast Gradient Sign Method (FGSM) attack [31] is widely studied, and the $\mathrm{C} \& \mathrm{~W}$ attack [22] is the most effective found so far on the application domains tested, mostly in computer vision. Both of these attack methods can be used for targeted or indiscriminate attacks.

With further examination of the attacker's capabilities, in addition to the potential control over the training data, a powerful attacker may also know the internal architecture and parameters of the classifier. Therefore, a fourth dimension can be added to the above taxonomy according to attacker information: in white-box attacks, the adversary generates malicious instances against the target classifier directly; while in black-box attacks, since the attacker does not possess full knowledge about the model, they first approximate the target's model by training over a dataset from a mixture of samples obtained by observing the target's performance, and synthetically generating inputs and label pairs. Then if the reconstructed model generalises well, the crafted adversarial examples against this model can be transferred to the target network and induce misclassifications. Papernot et al. [63, 62] have demonstrated the effectiveness of the black-box attack in certain specific domains. Specifically, they investigate intra- and cross-technique transferability between deep neural networks (DNNs), logistic regression, support vector machines (SVMs), decision trees and the $k$-nearest neighbour algorithm.

\subsection{Attacks against Reinforcement Learning}

In more recent studies, several papers have begun to study whether attacks against classifiers can also be applied to RL-based systems. Huang et al. [39] have shown that deep RL is vulnerable to adversarial samples generated by the 


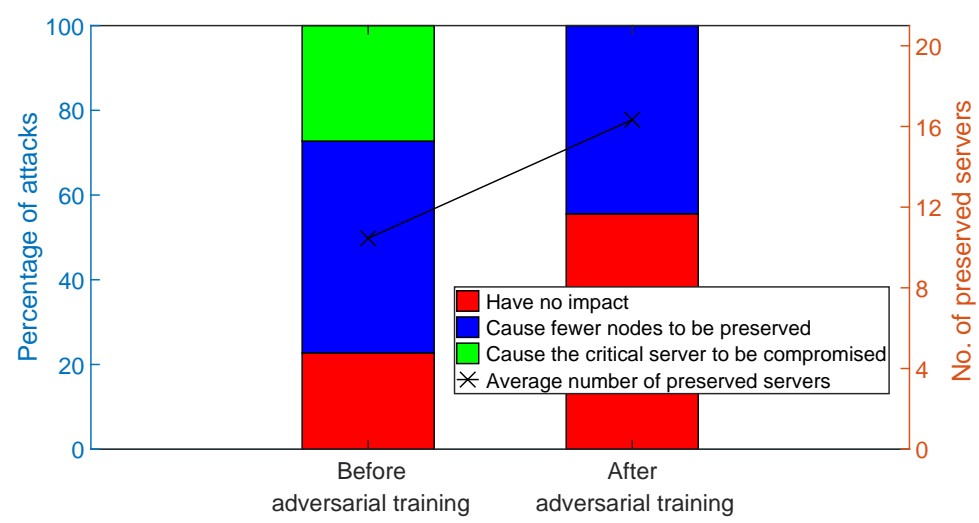

Fig. 8: Adversarial training against indiscriminate white-box attacks with limits on the choices of FPs and FNs (against DDQN), $F P \in\{18,23\}, F N \in\{12,19\}$

Fast Gradient Sign Method [31]. Their experimental results demonstrate that both white-box and black-box attacks are effective, even though the less knowledge the adversary has, the less effective the adversarial samples are.

Behzadan \& Munir [15] establish that adversaries can interfere with the training process of DQNs, preventing the victim from learning the correct policy. Specifically, the attacker applies minimum perturbation to the state observed by the target, so that a different action is chosen as the optimal action at the next state. The perturbation is generated using the same techniques proposed against DNN classifiers. In addition, the authors demonstrate the possibility of policy manipulation, where the victim ends up with choosing the actions selected by the adversarial policy.

Lin et al. [47] propose two kinds of attacks against deep reinforcement learning agents. In strategically-timed attacks, instead of crafting the state at each time step, the adversary identifies a subset of most vulnerable steps, and uses the $\mathrm{C} \& \mathrm{~W}$ attack [22] to perturb the corresponding states. In enchanting attacks, the adversary uses sampling to iteratively find a sequence of actions that will take the agent to the target state, and craft the current state so that the agent will follow the next required action.

\subsection{Adversarial Machine Learning Defences}

A number of countermeasures have been proposed since the discovery of adversarial samples. These can be roughly categorised into two classes: data-driven defences and learner robustification.

Data-driven Defences This class of defences are data driven - they either filter out the malicious data, inject adversarial samples into the training dataset, or manipulate features via projection. These approaches are akin to black-box defences since they make little to no use of the learner.

- Filtering instances. These counter-measures assume that the poisoning data in the training dataset or the adversarial samples against the test dataset either exhibit different statistical features, or follow a different distribution. Therefore, they propose to identify and filter out the injected/perturbed data. For example, Laishram and Phoha [42] design a method called Curie to protect SVM classifiers against poisoning attacks that flip labels in the training dataset. Steinhardt, Koh and Liang [72] study the worst-case loss of both data dependent and independent sanitisation methods. Metzen et al. [52] propose to train a detector network that takes input from intermediate layers of a classification network, and filters out adversarial samples. Feinman et al. [29] use (i) kernel density estimates in the feature space of a final hidden layer, and (ii) Bayesian neural network uncertainty estimates to detect the adversarial samples against DNNs. Li et al. [45] apply principal component analysis (PCA) on the convolutional layers of an original convolutional neural network $(\mathrm{CNN})$, and use the extracted statistics to train a cascade classifier that can distinguish between valid and adversarial data. 
Table 2: Taxonomy of attacks on machine learners, with representative past work. As the taxonomy was designed for supervised learners, we include attacks on reinforcement learning in Section 6.2

\begin{tabular}{|c|c|c|c|}
\hline & & Integrity & Availability \\
\hline \multirow[t]{2}{*}{ Causative } & $\begin{array}{l}\text { Targeted } \\
\text { (training set } \\
\text { manipulation for } \\
\text { specific errors) }\end{array}$ & $\begin{array}{l}\text { Rubinstein } \text { et al. [67]: boiling frog attacks against the PCA } \\
\text { anomaly detection algorithm; } \\
\text { Li et al. [44]: poison training data against collaborative } \\
\text { filtering systems; } \\
\text { Mei \& Zhu [50]: identify the optimal training set to } \\
\text { manipulate different machine learners; } \\
\text { Burkard \& Lagesse [19]: targeted causative attack on } \\
\text { Support Vector Machines that are learning from a data } \\
\text { stream }\end{array}$ & $\begin{array}{l}\text { Newsome } \text { et al. }[60] \text { : manipulate } \\
\text { training set of classifiers for worms } \\
\text { and spam to block legitimate } \\
\text { instances; } \\
\text { Chung \& Mok [24]: generate } \\
\text { harmful signatures to filter out } \\
\text { legitimate network traffic; } \\
\text { Nelson } \text { et al. [58]: exploit statistical } \\
\text { machine learning against a popular } \\
\text { email spam filter }\end{array}$ \\
\hline & $\begin{array}{l}\text { Indiscriminate } \\
\text { (training set } \\
\text { manipulation to } \\
\text { maximise overall } \\
\text { error rate) }\end{array}$ & $\begin{array}{l}\text { Biggio } \text { et al. }|18| \text { : inject crafted training data to increase } \\
\text { SVM's test error; } \\
\text { Xiao } \text { et al. }|78| \text { : label flips attack against SVMs; } \\
\text { Koh \& Liang [41]: minimise the number of crafted training } \\
\text { data via influence analysis }\end{array}$ & $\begin{array}{l}\text { Newsome } \text { et al. }[60] \text {; Chung \& Mok } \\
\text { [24]; Nelson } \text { et al. }[58]\end{array}$ \\
\hline \multirow[t]{2}{*}{ Exploratory } & $\begin{array}{l}\text { Targeted (test } \\
\text { instance } \\
\text { manipulation for } \\
\text { specific errors) }\end{array}$ & $\begin{array}{l}\text { Nelson et al. [59]: probe a classifier to determine good } \\
\text { attack points; } \\
\text { Papernot et al. [64]: exploits forward derivatives to search } \\
\text { for the minimum regions of the inputs to perturb; } \\
\text { Goodfellow et al. [31]: design the "fast gradient sign } \\
\text { method" (FGSM) to generate adversarial samples; } \\
\text { Carlini \& Wagner [22]: propose the C\&W method for } \\
\text { creating adversarial samples; } \\
\text { Han \& Rubinstein [32]: improve the gradient descent } \\
\text { method by replacing with gradient quotient }\end{array}$ & $\begin{array}{l}\text { Moore } \text { et al. }|55| \text { : provide } \\
\text { quantitative estimates of } \\
\text { denial-of-service activity }\end{array}$ \\
\hline & $\begin{array}{l}\text { Indiscriminate } \\
\text { (test instance } \\
\text { manipulation for } \\
\text { misclassifica- } \\
\text { tion) }\end{array}$ & $\begin{array}{l}\text { Biggio et al. [17]: use gradient descent method to find } \\
\text { attack instances against SVMs; } \\
\text { Szegedy et al. [74] demonstrate that changes imperceptible } \\
\text { to human eyes can make DNNs misclassify an image; } \\
\text { Goodfellow et al. [31]; } \\
\text { Papernot } \text { et al. [63, 62]: attack the target learner via a } \\
\text { surrogate model; } \\
\text { Moosavi-Dezfooli et al. [56] 57]: propose DeepFool that } \\
\text { generates universal perturbations to fool multiple DNNs; } \\
\text { Carlini \& Wagner [22]; } \\
\text { Nguyen } \text { et al. [61]: produce images that are unrecognisable } \\
\text { to humans, but can be recognised by DNNs; } \\
\text { Han \& Rubinstein [32] }\end{array}$ & Moore et al. [55] \\
\hline
\end{tabular}

- Injecting data. Goodfellow et al. [31] attribute the existence of adversarial samples to the "blind spots" of the training algorithm, and propose injecting adversarial examples into training, in order to improve the generalisation capabilities of DNNs [74, 31] - akin to active learning in non-adversarial settings. Tramer et al. [75] extend such adversarial training methods by incorporating perturbations generated against other models.

- Projecting data. Previous work has shown that high dimensionality facilitates the generation of adversarial samples, resulting in an increased attack surface. For example, Wang, Gao and Qi [76] theorise that a single unnecessary feature can ruin the robustness of a model. To counter this, data can be projected into lower-dimensional space before testing. Specifically, Bhagoji et al. [16] and Zhang et al. [80] propose defence methods based on dimensionality reduction via principal component analysis (PCA), and reduced feature sets, respectively. Das et al. [25] demonstrate that JPEG compression can be used as a pre-processing step to defend against evasion attacks in computer vision, potentially because JPEG compression removes high-frequency signal components, which helps 
remove imperceptible perturbations. However, these results contradict those obtained by Li and Vorobeychik [43], which suggests that more features should be used when facing adversarial evasion.

Learner Robustification Rather than focusing solely on training and test data, this class of methods-which are white-box in nature-aim to design models to be less susceptible to adversarial samples in the first place.

- Stabilisation. Zheng et al. [81] design stability training that modifies the model's objective function by adding a stability term. Their experimental results demonstrate that such a modification stabilises DNNs against small perturbations in the inputs. Papernot $e t$ al. [65] provide further examples using a distillation strategy against a saliency-map attack. However, this method has been shown to be ineffective by Carlini and Wagner [21]. Hosseini et al. [36] propose to improve adversarial training by adding an additional "NULL" class, and attempt to classify all adversarial samples as invalid.

- Moving target. Sengupta et al. [71] apply moving target defences against exploratory attacks: instead of using a single model for classification, the defender prepares a pool of models, and for each image to be classified, one trained DNN is picked following some specific strategy. The authors formulate the interaction as a Repeated Bayesian Stackelberg Game, and show that their approach can decrease the attack's success rate, while maintaining high accuracy for legitimate users.

- Robust statistics. Another avenue that has remained relatively unexplored is to leverage ideas from robust statistics, such as influence functions, break-down points, and $M$-estimators with robust loss functions (such as the Huber loss) that place diminishing cost to increasingly erroneous predictions. Rubinstein et al. [67] were the first to leverage robust statistics in adversarial learning settings for cyber-security, by applying a robust form of principal components analysis that optimises median absolute deviations instead of variance to defend against causative attacks on network-wide volume anomaly detection. Recently, interest in the theoretical computer science community has turned to robust estimation in high dimensions, e.g., Diakonikolas et al. [26].

Lessons Learned Despite many defences proposed, several recent studies [35, 23] point out that most of these methods (i) unrealistically assume that the attacker is not aware of the defence mechanism, and (ii) only consider relatively weak attacks, e.g., FGSM [31]. Negative results are reported on the effectiveness of these methods against adaptive attackers that are aware of the defence and act accordingly, and against the C\&W attack [22] (empirically the most efficient exploratory attack proposed so far).

Specifically, He et al. [35] demonstrate that the following recently proposed methods cannot defend against adaptive adversaries: (i) feature squeezing [79], (ii) specialists+1 ensemble method [10], and (iii) ensemble of methods in the papers [52, 29, 30]. Carlini and Wagner [23] show that ten detection methods for adversarial samples can be defeated, either by the $\mathrm{C} \& \mathrm{~W}$ attack [22], or white-box/black-box attacks. The authors conclude that the most effective defence so far for DNNs is to apply randomness to the DNN, because it makes generating adversarial samples against the target classifier as difficult as generating transferable adversarial samples. More recently, Athalye et al. [12] show that defences relied on obfuscated gradients can also be circumvented.

While most of the previous work on adversarial machine learning focused on the vision domain, this paper applies RL in cyber security, and studies how it reacts against different forms of causative integrity attacks.

\section{Conclusions and Future Work}

In this paper, we demonstrated the feasibility of developing autonomous defence in SDN using RL algorithms. In particular, we studied the impact of different forms of causative attacks, and showed that even though these attacks might cause RL agents to take sub-optimal actions, adversarial training could be applied to mitigate the impact.

For future work, we plan to (1) use a traffic generator to introduce background traffic between nodes, and use network performance metrics to replace the current binary states; (2) consider different types of network traffic, so that the actions of the RL agent could include partial isolation in terms of blocking certain protocols between nodes; (3) change full observability of the network status to partial observability-the defender may have limited resources, and the attacker may not know the entire topology; and (4) remove limiting assumptions, e.g., the attacker having to compromise all nodes along the path to the critical server. 


\section{Bibliography}

[1] Amazon EC2 Instance Types - Amazon Web Services (AWS), https://aws.amazon.com/ec2/ instance-types/

[2] OpenFlow. http://archive.openflow.org/wp/learnmore/ (2011)

[3] SDN architecture. Tech. rep. (Jun 2014), https://www.opennetworking.org/wp-content/ uploads/2013/02/TR_SDN_ARCH_1.0_06062014.pdf

[4] Open vSwitch. http://openvswitch.org/ (2016)

[5] Cisco Open SDN Controller. https://www.cisco.com/c/en/us/products/cloud-systems-management/open-sdncontroller/index.html (2017)

[6] Floodlight OpenFlow Controller. http://www.projectfloodlight.org/floodlight/ (2017)

[7] Mininet: An Instant Virtual Network on your Laptop. http://mininet.org/ (2017)

[8] NOX/POX. https://github.com/noxrepo (2017)

[9] OpenDaylight. https://www.opendaylight.org/ (2017)

[10] Abbasi, M., Gagné, C.: Robustness to Adversarial Examples through an Ensemble of Specialists. eprint arXiv:1702.06856 (2017), http://arxiv.org/abs/1702.06856

[11] Alpcan, T., Baar, T.: Network Security: A Decision and Game-Theoretic Approach. Cambridge University Press, New York, NY, USA, 1st edn. (2011)

[12] Athalye, A., Carlini, N., Wagner, D.: Obfuscated Gradients Give a False Sense of Security: Circumventing Defenses to Adversarial Examples. arXiv:1802.00420 [cs] (Feb 2018), http://arxiv.org/abs/1802. 00420 , arXiv: 1802.00420

[13] Barreno, M., Nelson, B., Joseph, A.D., Tygar, J.D.: The Security of Machine Learning. Machine Learning 81(2), 121-148 (Nov 2010), https://doi.org/10.1007/s10994-010-5188-5

[14] Beaudoin, L.: Autonomic computer network defence using risk states and reinforcement learning. Ph.D. thesis, University of Ottawa (Canada) (2009)

[15] Behzadan, V., Munir, A.: Vulnerability of Deep Reinforcement Learning to Policy Induction Attacks. eprint arXiv:1701.04143 (2017), https://arxiv.org/abs/1701.04143

[16] Bhagoji, A.N., Cullina, D., Mittal, P.: Dimensionality Reduction as a Defense against Evasion Attacks on Machine Learning Classifiers. arXiv:1704.02654 (2017), https://arxiv.org/abs/1704.02654

[17] Biggio, B., Corona, I., Nelson, B., Rubinstein, B.I., Maiorca, D., Fumera, G., Giacinto, G., Roli, F.: Security evaluation of support vector machines in adversarial environments. In: Support Vector Machines Applications, pp. 105-153. Springer International Publishing (2014), https://arxiv.org/abs/1401.7727

[18] Biggio, B., Nelson, B., Laskov, P.: Poisoning Attacks against Support Vector Machines. In: Proceedings of the 29th International Coference on International Conference on Machine Learning. pp. 1467-1474. Omnipress, Edinburgh, Scotland (2012), http://arxiv.org/abs/1206.6389

[19] Burkard, C., Lagesse, B.: Analysis of Causative Attacks Against SVMs Learning from Data Streams. In: Proceedings of the 3rd ACM on International Workshop on Security And Privacy Analytics. pp. 31-36. IWSPA '17, ACM, New York, NY, USA (2017), http://doi.acm.org/10.1145/3041008.3041012

[20] Cao, S., Wang, Y., Xu, C.: Service Migrations in the Cloud for Mobile Accesses: A Reinforcement Learning Approach. In: Proceedings of the 2017 International Conference on Networking, Architecture, and Storage (NAS). pp. 1-10 (Aug 2017)

[21] Carlini, N., Wagner, D.: Defensive Distillation is Not Robust to Adversarial Examples. arXiv:1607.04311 (2016)

[22] Carlini, N., Wagner, D.: Towards Evaluating the Robustness of Neural Networks. eprint arXiv:1608.04644 (2016), http://arxiv.org/abs/1608.04644, arXiv: 1608.04644

[23] Carlini, N., Wagner, D.: Adversarial Examples Are Not Easily Detected: Bypassing Ten Detection Methods. eprint arXiv:1705.07263 (2017), http: //arxiv.org/abs/1705.07263, arXiv: 1705.07263

[24] Chung, S.P., Mok, A.K.: Advanced Allergy Attacks: Does a Corpus Really Help? In: Recent Advances in Intrusion Detection. pp. 236-255. Lecture Notes in Computer Science, Springer, Berlin, Heidelberg (Sep 2007), https://link.springer.com/chapter/10.1007/978-3-540-74320-0_13 
[25] Das, N., Shanbhogue, M., Chen, S.T., Hohman, F., Chen, L., Kounavis, M.E., Chau, D.H.: Keeping the Bad Guys Out: Protecting and Vaccinating Deep Learning with JPEG Compression. eprint arXiv:1705.02900 (May 2017), http://arxiv.org/abs/1705.02900, arXiv: 1705.02900

[26] Diakonikolas, I., Kamath, G., Kane, D.M., Li, J., Moitra, A., Stewart, A.: Robust Estimators in High Dimensions without the Computational Intractability. In: Proceedings of the 2016 IEEE 57th Annual Symposium on Foundations of Computer Science (FOCS). pp. 655-664 (Oct 2016)

[27] Duggan, M., Duggan, J., Howley, E., Barrett, E.: A reinforcement learning approach for the scheduling of live migration from under utilised hosts. Memetic Computing pp. 1-11 (Dec 2016), https:// link . springer. com/article/10.1007/s12293-016-0218-x

[28] Everitt, T., Krakovna, V., Orseau, L., Hutter, M., Legg, S.: Reinforcement Learning with a Corrupted Reward Channel. eprint arXiv:1705.08417 (2017), http://arxiv.org/abs/1705.08417

[29] Feinman, R., Curtin, R.R., Shintre, S., Gardner, A.B.: Detecting Adversarial Samples from Artifacts. eprint arXiv:1703.00410 (2017), http: //arxiv.org/abs/1703.00410, arXiv: 1703.00410

[30] Gong, Z., Wang, W., Ku, W.S.: Adversarial and Clean Data Are Not Twins. eprint arXiv:1704.04960 (2017), http://arxiv.org/abs/1704.04960

[31] Goodfellow, I.J., Shlens, J., Szegedy, C.: Explaining and Harnessing Adversarial Examples. eprint arXiv:1412.6572 (2014), http://arxiv.org/abs/1412.6572

[32] Han, Y., Rubinstein, B.I.P.: Adequacy of the Gradient-Descent Method for Classifier Evasion Attacks. arXiv:1704.01704 (Apr 2017), http: //arxiv.org/abs/1704.01704, arXiv: 1704.01704

[33] Hasselt, H.V.: Double Q-learning. In: Lafferty, J.D., Williams, C.K.I., Shawe-Taylor, J., Zemel, R.S., Culotta, A. (eds.) Advances in Neural Information Processing Systems 23, pp. 2613-2621. Curran Associates, Inc. (2010), http://papers.nips.cc/paper/3964-double-q-learning.pdf

[34] Hasselt, H.V., Guez, A., Silver, D.: Deep Reinforcement Learning with Double Q-learning. eprint arXiv:1509.06461 (Sep 2015), http://arxiv.org/abs/1509.06461, arXiv: 1509.06461

[35] He, W., Wei, J., Chen, X., Carlini, N., Song, D.: Adversarial Example Defenses: Ensembles of Weak Defenses are not Strong. eprint arXiv:1706.04701 (2017), http://arxiv.org/abs/1706.04701, arXiv: 1706.04701

[36] Hosseini, H., Chen, Y., Kannan, S., Zhang, B., Poovendran, R.: Blocking Transferability of Adversarial Examples in Black-Box Learning Systems. eprint arXiv:1703.04318 (2017), http://arxiv.org/abs/1703. 04318, arXiv: 1703.04318

[37] Huang, L., Joseph, A.D., Nelson, B., Rubinstein, B.I., Tygar, J.: Adversarial machine learning. In: Proceedings of the 4th ACM Workshop on Security and Artificial Intelligence. pp. 43-58. ACM (2011)

[38] Huang, R., Chu, X., Zhang, J., Hu, Y.H.: Energy-efficient Monitoring in Software Defined Wireless Sensor Networks Using Reinforcement Learning: A Prototype. Int. J. Distrib. Sen. Netw. 2015, 1:1-1:1 (Jan 2015), https://doi.org/10.1155/2015/360428

[39] Huang, S., Papernot, N., Goodfellow, I., Duan, Y., Abbeel, P.: Adversarial Attacks on Neural Network Policies. eprint arXiv:1702.02284 (2017), https://arxiv.org/abs/1702.02284

[40] Kim, S., Son, J., Talukder, A., Hong, C.S.: Congestion prevention mechanism based on Q-leaning for efficient routing in SDN. In: Proceedings of the 2016 International Conference on Information Networking (ICOIN). pp. 124-128 (Jan 2016)

[41] Koh, P.W., Liang, P.: Understanding Black-box Predictions via Influence Functions. arXiv:1703.04730 [cs, stat] (Mar 2017), http://arxiv.org/abs/1703.04730, arXiv: 1703.04730

[42] Laishram, R., Phoha, V.V.: Curie: A method for protecting SVM Classifier from Poisoning Attack. arXiv:1606.01584 [cs] (Jun 2016), http://arxiv.org/abs/1606.01584, arXiv: 1606.01584

[43] Li, B., Vorobeychik, Y.: Feature Cross-substitution in Adversarial Classification. In: Proceedings of the 2014 NIPS. pp. 2087-2095. NIPS'14, MIT Press, Cambridge, MA, USA (2014), http://dl.acm.org/ Citation.cfm?id=2969033.2969060

[44] Li, B., Wang, Y., Singh, A., Vorobeychik, Y.: Data Poisoning Attacks on Factorization-Based Collaborative Filtering. eprint arXiv:1608.08182 (2016), http://adsabs . harvard.edu/abs/2016arXiv160808182L

[45] Li, X., Li, F.: Adversarial Examples Detection in Deep Networks with Convolutional Filter Statistics. arXiv:1612.07767 [cs] (Dec 2016), http: / /arxiv.org/abs/1612.07767, arXiv: 1612.07767

[46] Lin, S.C., Akyildiz, I.F., Wang, P., Luo, M.: QoS-Aware Adaptive Routing in Multi-layer Hierarchical Software Defined Networks: A Reinforcement Learning Approach. In: Proceedings of the 2016 IEEE International Conference on Services Computing (SCC). pp. 25-33 (Jun 2016) 
[47] Lin, Y.C., Hong, Z.W., Liao, Y.H., Shih, M.L., Liu, M.Y., Sun, M.: Tactics of Adversarial Attack on Deep Reinforcement Learning Agents. eprint arXiv:1703.06748 (Mar 2017), http://arxiv.org/abs/1703. 06748, arXiv: 1703.06748

[48] Mao, H., Alizadeh, M., Menache, I., Kandula, S.: Resource Management with Deep Reinforcement Learning. In: Proceedings of the 15th ACM Workshop on Hot Topics in Networks. pp. 50-56. HotNets'16, ACM, New York, NY, USA (2016), http://doi.acm.org/10.1145/3005745.3005750

[49] Medved, J., Varga, R., Tkacik, A., Gray, K.: OpenDaylight: Towards a Model-Driven SDN Controller architecture. In: Proceeding of IEEE International Symposium on a World of Wireless, Mobile and Multimedia Networks 2014. pp. 1-6 (Jun 2014)

[50] Mei, S., Zhu, X.: Using Machine Teaching to Identify Optimal Training-set Attacks on Machine Learners. In: Proceedings of the Twenty-Ninth AAAI Conference on Artificial Intelligence. pp. 2871-2877. AAAI' 15, AAAI Press, Austin, Texas (2015), http://dl.acm.org/citation.cfm?id=2886521.2886721

[51] Mestres, A., Rodriguez-Natal, A., Carner, J., Barlet-Ros, P., Alarcón, E., Solé, M., Muntés-Mulero, V., Meyer, D., Barkai, S., Hibbett, M.J., Estrada, G., Ma'ruf, K., Coras, F., Ermagan, V., Latapie, H., Cassar, C., Evans, J., Maino, F., Walrand, J., Cabellos, A.: Knowledge-Defined Networking. SIGCOMM Comput. Commun. Rev. 47(3), 2-10 (Sep 2017), http://doi.acm.org/10.1145/3138808.3138810

[52] Metzen, J.H., Genewein, T., Fischer, V., Bischoff, B.: On Detecting Adversarial Perturbations. eprint arXiv:1702.04267 (2017), http://arxiv.org/abs/1702.04267, arXiv: 1702.04267

[53] Mnih, V., Badia, A.P., Mirza, M., Graves, A., Harley, T., Lillicrap, T.P., Silver, D., Kavukcuoglu, K.: Asynchronous Methods for Deep Reinforcement Learning. In: Proceedings of the 33rd International Conference on International Conference on Machine Learning - Volume 48. pp. 1928-1937. ICML'16, JMLR.org, New York, NY, USA (2016), http://dl.acm.org/citation.cfm?id=3045390.3045594

[54] Mnih, V., Kavukcuoglu, K., Silver, D., Graves, A., Antonoglou, I., Wierstra, D., Riedmiller, M.A.: Playing Atari with Deep Reinforcement Learning. CoRR abs/1312.5602 (2013), http://arxiv.org/abs/1312.5602

[55] Moore, D., Shannon, C., Brown, D.J., Voelker, G.M., Savage, S.: Inferring Internet Denial-of-service Activity. ACM Trans. Comput. Syst. 24(2), 115-139 (May 2006), http://doi.acm.org/10.1145/1132026. 1132027

[56] Moosavi-Dezfooli, S.M., Fawzi, A., Fawzi, O., Frossard, P.: Universal Adversarial Perturbations. eprint arXiv:1610.08401 (2016), http://arxiv.org/abs/1610.08401

[57] Moosavi-Dezfooli, S.M., Fawzi, A., Frossard, P.: DeepFool: A Simple and Accurate Method to Fool Deep Neural Networks. In: CVPR. pp. 2574-2582 (2016), https://arxiv.org/abs/1511.04599

[58] Nelson, B., Barreno, M., Chi, F.J., Joseph, A.D., Rubinstein, B.I., Saini, U., Sutton, C.A., Tygar, J.D., Xia, K.: Exploiting Machine Learning to Subvert Your Spam Filter. In: Proceedings of the First USENIX Workshop on Large-scale Exploits and Emergent Threats (LEET'08) (2008), http://blaine-nelson.com/ research/pubs/Nelson-Barreno-LEET-2008.pdf

[59] Nelson, B., Rubinstein, B.I., Huang, L., Joseph, A.D., Lee, S.J., Rao, S., Tygar, J.: Query strategies for evading convex-inducing classifiers. Journal of Machine Learning Research 13(May), 1293-1332 (2012), http: //www.jmlr.org/papers/v13/nelson12a.html

[60] Newsome, J., Karp, B., Song, D.: Paragraph: Thwarting Signature Learning by Training Maliciously. In: Proceedings of the 9th International Conference on Recent Advances in Intrusion Detection. pp. 81-105. RAID'06, Springer-Verlag, Berlin, Heidelberg (2006), http://dx.doi.org/10.1007/11856214_5

[61] Nguyen, A., Yosinski, J., Clune, J.: Deep Neural Networks are Easily Fooled: High Confidence Predictions for Unrecognizable Images. In: CVPR. pp. 427-436 (2015), https: / /arxiv.org/abs/1412.1897

[62] Papernot, N., McDaniel, P., Goodfellow, I.: Transferability in Machine Learning: from Phenomena to Black-Box Attacks using Adversarial Samples. eprint arXiv:1605.07277 (2016), http://arxiv.org/abs/1605. 07277

[63] Papernot, N., McDaniel, P., Goodfellow, I., Jha, S., Celik, Z.B., Swami, A.: Practical Black-Box Attacks against Deep Learning Systems using Adversarial Examples. eprint arXiv:1602.02697 (2016), https://arxiv. org/abs/1602.02697v3

[64] Papernot, N., McDaniel, P., Jha, S., Fredrikson, M., Celik, Z.B., Swami, A.: The Limitations of Deep Learning in Adversarial Settings. In: Proceedings of the European Symposium on Security \& Privacy. pp. 372-387 (2016), https://arxiv.org/abs/1511.07528 
[65] Papernot, N., McDaniel, P., Wu, X., Jha, S., Swami, A.: Distillation as a Defense to Adversarial Perturbations against Deep Neural Networks. eprint arXiv:1511.04508 (2015), http://arxiv.org/abs/1511.04508. arXiv: 1511.04508

[66] Pinto, L., Davidson, J., Sukthankar, R., Gupta, A.: Robust Adversarial Reinforcement Learning. eprint arXiv:1703.02702 (2017), https://arxiv.org/abs/1703.02702

[67] Rubinstein, B.I., Nelson, B., Huang, L., Joseph, A.D., Lau, S.h., Rao, S., Taft, N., Tygar, J.: ANTIDOTE: Understanding and defending against poisoning of anomaly detectors. In: Proceedings of the 9th ACM SIGCOMM Conference on Internet Measurement. pp. 1-14. ACM (2009), https://people.eng.unimelb.edu. au/brubinstein/papers/imc206-rubinstein.pdf

[68] Salahuddin, M.A., Al-Fuqaha, A., Guizani, M.: Reinforcement learning for resource provisioning in the vehicular cloud. IEEE Wireless Communications 23(4), 128-135 (Aug 2016)

[69] Salahuddin, M.A., Al-Fuqaha, A., Guizani, M.: Software-Defined Networking for RSU Clouds in Support of the Internet of Vehicles. IEEE Internet of Things Journal 2(2), 133-144 (Apr 2015), http: / / ieeexplore. ieee.org/abstract/document/6949072/

[70] Schaul, T., Quan, J., Antonoglou, I., Silver, D.: Prioritized Experience Replay. CoRR abs/1511.05952 (2015), http://arxiv.org/abs/1511.05952

[71] Sengupta, S., Chakraborti, T., Kambhampati, S.: Securing Deep Neural Nets against Adversarial Attacks with Moving Target Defense. eprint arXiv:1705.07213 (May 2017), http://arxiv.org/abs/1705.07213. arXiv: 1705.07213

[72] Steinhardt, J., Koh, P.W., Liang, P.: Certified Defenses for Data Poisoning Attacks. eprint arXiv:1706.03691 (Jun 2017), http://arxiv.org/abs/1706.03691, arXiv: 1706.03691

[73] Sutton, R.S., Barto, A.G.: Introduction to Reinforcement Learning. MIT Press, Cambridge, MA, USA, first edn. (1998)

[74] Szegedy, C., Zaremba, W., Sutskever, I., Bruna, J., Erhan, D., Goodfellow, I., Fergus, R.: Intriguing Properties of Neural Networks. eprint arXiv:1312.6199 (2013), http: //arxiv.org/abs/1312.6199

[75] Tramèr, F., Kurakin, A., Papernot, N., Boneh, D., McDaniel, P.: Ensemble Adversarial Training: Attacks and Defenses. eprint arXiv:1705.07204 (May 2017), http://arxiv.org/abs/1705.07204, arXiv: 1705.07204

[76] Wang, B., Gao, J., Qi, Y.: A Theoretical Framework for Robustness of (Deep) Classifiers against Adversarial Examples. eprint arXiv:1612.00334 (2016), http: / / arxiv.org/abs/1612.00334, arXiv: 1612.00334

[77] Wang, Z., Chen, C., Li, H.X., Dong, D., Tarn, T.J.: A novel incremental learning scheme for reinforcement learning in dynamic environments. In: Proceedings of the 12th World Congress on Intelligent Control and Automation (WCICA). pp. 2426-2431 (Jun 2016)

[78] Xiao, H., Xiao, H., Eckert, C.: Adversarial Label Flips Attack on Support Vector Machines. In: Proceedings of the 20th European Conference on Artificial Intelligence. pp. 870-875. ECAI'12, IOS Press, Amsterdam, The Netherlands, The Netherlands (2012), https://doi.org/10.3233/978-1-61499-098-7-870

[79] Xu, W., Evans, D., Qi, Y.: Feature Squeezing: Detecting Adversarial Examples in Deep Neural Networks. eprint arXiv:1704.01155 (2017), http://arxiv.org/abs/1704.01155

[80] Zhang, F., Chan, P.P.K., Biggio, B., Yeung, D.S., Roli, F.: Adversarial Feature Selection Against Evasion Attacks. IEEE Transactions on Cybernetics 46(3), 766-777 (Mar 2016), http: / / ieeexplore. ieee.org/ document/7090993/

[81] Zheng, S., Song, Y., Leung, T., Goodfellow, I.: Improving the Robustness of Deep Neural Networks via Stability Training. eprint arXiv:1604.04326 (2016), https://arxiv.org/abs/1604.04326 